\title{
Severe bronchospasm in a premature infant during induction of anesthesia caused ventilation failure
}

\author{
Yoon Ji Choi ${ }^{1}$, Sung-Uk Choi ${ }^{2}$, Eun-Jung $\mathrm{Cho}^{2}$, Jae Yoon $\mathrm{Oh}^{1}$, and Hae-Ja Lim ${ }^{2}$ \\ Department of Anesthesiology and Pain Medicine, ${ }^{1}$ Seoul Paik Hospital, College of Medicine, Inje University, ${ }^{2}$ College of Medicine, \\ Korea University, Seoul, Korea
}

A 3-month-old (39 weeks postconceptual age) male infant weighing $2.9 \mathrm{~kg}$ was scheduled for laser photocoagulation with a diagnosis of retinopathy of prematurity. He was born at 27 weeks gestation with a birth weight of $970 \mathrm{~g}$. He had been mechanically ventilated from birth for 20 days for respiratory insufficiency due to respiratory distress syndrome (RDS) and bronchopulmonary dysplasia (BPD). A chest X-ray performed before the subsequent ligation of the patent ductus arteriosus showed bilateral haziness in the entire lung field due to the RDS and BPD during tracheal intubation. The infant required ventilation with a high concentration of oxygen and received surfactant therapy.

Twenty days after birth, patent ductus arteriosus (PDA) ligation was done under general anesthesia in which induction was achieved with inhalation of sevoflurane and $1 \mathrm{mg}$ of rocuronium. The operation proceeded uneventfully and the extubation was performed five days after the surgery. After extubation, the infant was able to breathe spontaneously with an incubator oxygen supply, and the oxygen saturation was maintained above $90 \%$.

After PDA ligation, the infant was diagnosed with retinopathy of prematurity, and he was then scheduled for laser photocoagulation. On the chest X-ray, improvement of haziness was observed from five days before the operation. After consultation with pediatrics, the decision was made to operate.

Upon arrival in the operating room, electrocardiography, pulse oximetry, and noninvasive blood pressure were monitored, and the patient's vital signs were stable. Induction of anesthesia was achieved with thiopental (15 $\mathrm{mg})$, rocuronium $(2 \mathrm{mg})$, and sevoflurane. The tracheal intubation was performed with an uncuffed $3.5 \mathrm{~mm}$ internal diameter endotracheal tube, but there was no capnogram trace after three breaths. At this time, the oxygen saturation rapidly dropped to below $80 \%$. The endotracheal tube was removed because the anesthesiologist suspected esophageal intubation and the patient was ventilated with $100 \%$ oxygen via face mask. Mask ventilation was not performed well, and peak inspiratory pressure was revealed to be above 25 $\mathrm{mmHg}$. The anesthesiologist suspected stiff lungs, which suggested bronchospasm. Hydrocortisone sodium succinate (SoluCortef $^{\circledR}$, Pfizer Inc., New York, NY, USA) 20 mg was intravenously injected. Five minutes later, oxygen saturation slowly increased up to $99 \%$ and reintubation was attempted. Although it was confirmed by direct laryngoscopy that the tube had passed between the vocal cords, proper ventilation of the lungs was not achieved. No expired carbon dioxide was noted on the capnograph, and chest auscultation was equivocal. The oxygen saturation was then in the low sixties. After sevoflurane was administered by inhalation, a slight chest movement was noted, and oxygen saturation increased up to $80 \%$. During that time, a portable chest radiograph was taken which revealed severe consolidation with air bronchograms (Fig. 1A). Despite ventilatory support for 10 minutes, the oxygen saturation failed to increase beyond $80 \%$. Hydrocortisone sodium succinate $20 \mathrm{mg}$ was then intravenously injected once more. Afterwards, $\mathrm{SaO}_{2}$ was maintained at $88-93 \%$. The surgery was cancelled, and glycopyrrolate

Corresponding author: Sung-Uk Choi, M.D., Ph.D., Department of Anesthesiology and Pain Medicine, College of Medicine, Korea University, 5, Anam-dong, Sungbuk-gu, Seoul 136-705, Korea. Tel: 82-2-920-5632, Fax: 82-2-928-2275, E-mail: drchois@korea.ac.kr

(c) This is an open-access article distributed under the terms of the Creative Commons Attribution Non-Commercial License (http:// creativecommons.org/licenses/by-nc/3.0/), which permits unrestricted non-commercial use, distribution, and reproduction in any medium, provided the original work is properly cited. 

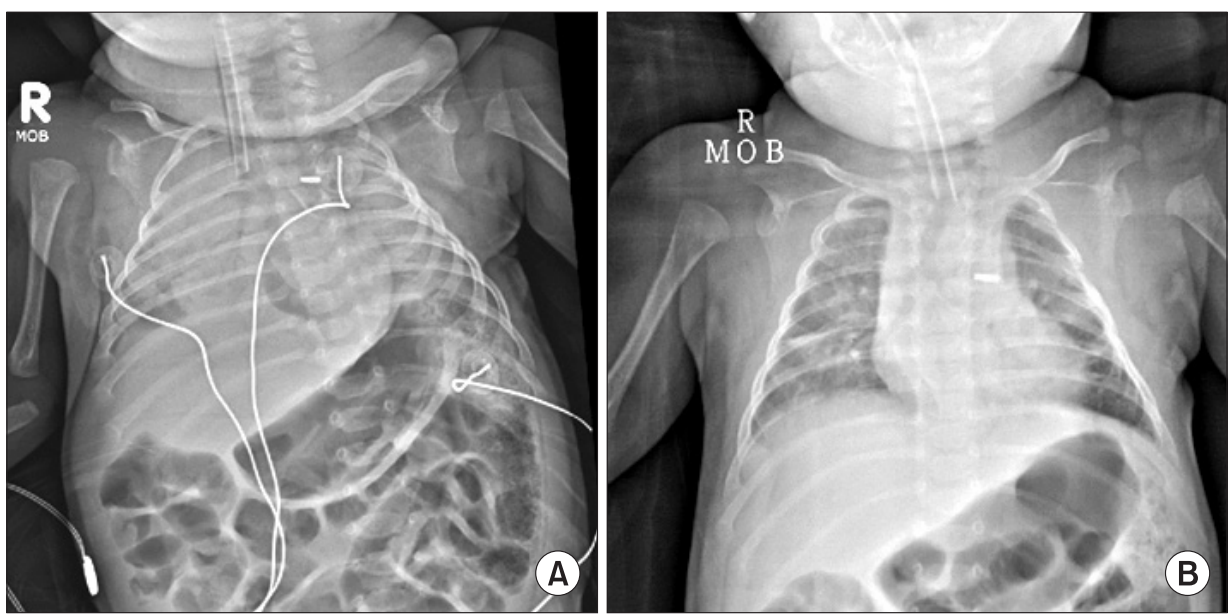

Fig. 1. (A) Chest X-ray taken immediately after the event showed severe bilateral bronchospasm with ill-defined haziness in both lungs. Consolidation with air bronchograms in right lung rules out atelectasis or pneumonia. Large amount of air in stomach and bowel. (B) Chest X-ray taken one hour later showed complete recovery of bronchospasm. Improved consolidation in right lung with remaining subsegmental atelectasis in right middle lung field.

$0.2 \mathrm{mg}$ and pyridostigmine $0.2 \mathrm{mg}$ were intravenously administered. The intubated patient was transferred to the neonatal intensive care unit (NICU) with Ambu bag ventilation for further management. While in transit to the NICU, the patient's vital signs were stable, and oxygen saturation was maintained above $90 \%$.

In the NICU, another chest radiograph was taken after the patient was stabilized, and this showed an improved state of consolidation (Fig. 1B). After the patient's spontaneous breathing completely returned and became regular, extubation was performed. The patient recovered without complications.

We experienced a case in which severe bronchospasm caused ventilation failure in a 3-month-old premature pediatric patient with a previous history of mechanical ventilation and incompletely resolved consolidation with underlying bronchopulmonary dysplasia. In this case, the onset of symptoms was rapid, including extreme stiffness of the lungs, and there was an absence of the end-tidal carbon dioxide trace noted immediately after intubation. During the event, the chest radiography revealed severe consolidation with air bronchograms.

Fatal contraction of airway smooth muscle can be observed spontaneously or by agonist. Thus, even within the first days of life, bronchospasm can occur in preterm infants [1].

Bronchospasm associated with anesthesia presents with an expiratory wheeze with/without auscultation, prolonged expiration and rising end-tidal carbon dioxide, increasing circuit pressure, increased pressures of inflation during positive pressure ventilation, and/or desaturation [2].

Wheeze cannot be audible either with or without auscultation because it is caused by gas flow in the patient's airways. Thus, in cases of severe bronchospasm such as our case, the chest may be silent or equivocal on auscultation and the diagnosis may be delayed on appropriate assessment of increased inflation pressures. However, wheeze may occur as bronchospasm but can be heard with a misplaced endotracheal tube, such as in bronchial or esophageal intubation, and with acute respiratory distress syndrome or pulmonary edema. We also make a wrong diagnosis because the patient had no detectable end-tidal carbon dioxide or chest movement. Thus, extubation was conducted and optimal management was delayed. In the same way, wheezing was not a typical symptom for diagnosis of bronchospasm, and the wheezing of our patient was not distinct because the patient underwent severe airway obstruction.

Increased inspiratory pressures may develop not only with bronchospasm but also with obstruction of a natural airway or any component of a breathing circuit and decreased compliance of the lungs or chest such as atelectasis, hemo-pneumothorax, or fentanyl induced rigidity [2]. Accordingly, the anesthesiologist takes a systematic approach to the diagnosis and treatment of bronchospasm in relation to anesthesia.

In our case, airway irritation from direct laryngoscopy and passage of the endotracheal tube is suspected to have caused bronchospasm during intubation. This procedure is noted for profoundly painful stimuli and is related to bronchospasm and/ or laryngospasm. In particular, a patient who has a previous respiratory history is closely connected to an increased risk for perioperative desaturation, bronchospasm, laryngospasm, or airway obstruction [3]. Furthermore, our patient underwent long-term mechanical ventilation. Therapy with high inspired oxygen concentrations or mechanical ventilation with high positive airway pressures leads to inflammation, fibrosis, or smooth muscle hypertrophy in the airways of premature infants [4], which might result in our patient having an increased vulnerability to bronchospasm.

We suggest that the anesthesiologist should carefully consider respiratory complications such as bronchospasm during the induction of anesthesia with a premature infant who has a previous history of mechanical ventilation and underlying respiratory diseases. In addition, we keep in mind the respiratory complications throughout general anesthesia. 


\section{References}

1. Sward-Comunelli SL, Mabry SM, Truog WE, Thibeault DW. Airway muscle in preterm infants: changes during development. J Pediatr 1997; 130: 570-6.

2. Westhorpe RN, Ludbrook GL, Helps SC. Crisis management during anaesthesia: bronchospasm. Qual Saf Health Care 2005 ; 14 : e7.

3. von Ungern-Sternberg BS, Boda K, Chambers NA, Rebmann C, Johnson C, Sly PD, et al. Risk assessment for respiratory complications in paediatric anaesthesia: a prospective cohort study. Lancet 2010; 376: 773-83.

4. O'Brodovich HM, Mellins RB. Bronchopulmonary dysplasia. Unresolved neonatal acute lung injury. Am Rev Respir Dis 1985; 132: 694-709. 